\title{
Improving diagnosis and prediction of progression in Mild Cognitive Impairment
}

\author{
Naaheed Mukadam, UCL Division of Psychiatry
}

Mild Cognitive Impairment $(\mathrm{MCl})$ is a clinical condition conceptualised as a stage between normal cognition and dementia. To diagnose it requires subjective cognitive impairment, evidence of cognitive impairment on cognitive testing but no abnormality in a person's functioning and no evidence of dementia (American Psychiatric Association, 2013). There has been growing interest in the condition over the past two decades or so because people with $\mathrm{MCl}$ are much more likely than people with no cognitive impairment to progress to dementia (Roberts et al., 2013). However, a significant percentage of people with $\mathrm{MCl}$ will not progress to dementia and some will revert to having normal cognition. Rates of progression and reversion to normal cognition vary widely in different studies (Manly et al., 2008). People with $\mathrm{MCl}$ experience worry about their symptoms and this is partly alleviated by receiving a diagnosis of $\mathrm{MCl}$ and being reassured they do not have dementia (Gomersall et al., 2017). The benefits of diagnosis also include gaining a greater understanding of their symptoms and accessing clinical support but a significant amount of uncertainty remains with regards to risk of progression and recipients of the diagnosis remain frustrated at the lack of treatments for $\mathrm{MCl}$ (Gomersall et al., 2017). There has been much interest in improving prediction of progression to dementia from $\mathrm{MCl}$ but to date, the best predictors of progression remain structured clinical and functional assessments, with some additional benefit from measures of cortical volume/thickness from brain imaging (Korolev et al., 2016). As yet, however, there are no interventions that can prevent (Kane et al., 2017) or treat (Cooper et al., 2013) $\mathrm{MCl}$ so it seems set to remain an important clinical entity for the foreseeable future.

Priorities for $\mathrm{MCl}$ research are to improve the accuracy and timeliness of diagnosis as well as the quality of monitoring and ability to predict progression to dementia or reversion to normal cognition. In this issue of International Psychogeriatrics we present three papers on this theme.

Any monitoring or treatment of $\mathrm{MCl}$ must start with a diagnosis of $\mathrm{MCl}$ with cognitive testing and enquiries about function. It is self-evident that if a function is not asked about then impairment in performing that function will not be identified and the diagnosis of $\mathrm{MCl}$ may be made when the person has dementia. It is therefore of paramount importance that assessment of a person's functioning be as sensitive as possible in order to make diagnosis and monitoring of change as accurate as possible. One aspect of functioning that may be neglected in history taking is the use of everyday technologies (ETs), which is the broad term for a wide range of technical objects and services that are used in our homes or the community, such as cash machines, coffee makers and cell phones. Many of these technologies can require quite complex cognitive functions, and difficulties in using ETs has been shown to be a marker for cognitive difficulties (Malinowsky et al., 2010). Hedman et al (2018) explore how the use of ETs changes over time and if difficulty with particular ETs was more sensitive to cognitive decline. The authors recruited participants over the 
age of 55 years who were diagnosed with $\mathrm{MCl}$ at a specialised memory clinic and compared their perceived ability to use ETs at baseline and after five years. They used the Everyday Technology Use Questionnaire (ETUQ) which is a structured questionnaire that has been validated in older adults with cognitive impairments and includes 92 ETs that are commonly used by older people inside and outside the home (Rosenberg et al., 2009). Participants rated their ability to use the ETs on a Likert scale. Around $70 \%$ of those approached initially agreed to participate, $62 \%$ of the sample converted to dementia over 5 years and there was a $43 \%$ drop-out rate after five years. Overall, participants' ability to use ETs decreased although $85 \%$ of ETs retained the same overall perceived level of difficulty of use. ETs that were rated as relatively more challenging than other technology at baseline were stereo/CD players, entryphones, digital TV receivers, digital cameras, internet banking, remote controls and cell phones. However, out of these, only CD players and digital cameras were significantly more challenging to use after five years than at baseline. Out of technologies rated as relatively less challenging to use at baseline, only washing machines, TV with remote controls, hand-held mixers and internet interactions were rated as more challenging at five years compared to baseline. This study highlights the importance of considering a wide range of activities as well as technologies when assessing for functional impairment accompanying cognitive impairment. It also highlights certain common ETs that it would be useful to focus on when conducting clinical assessments, in order to improve sensitivity to functional change.

Screening tools for $\mathrm{MCl}$ are a useful adjunct for a clinical diagnosis in terms of having a baseline score which can support the diagnosis and be used for monitoring. Park et al (2018) describe and validate a new computerised test for $\mathrm{MCl}$ screening. The authors initially conducted a systematic review of screening test systems for $\mathrm{MCl}$ and removed duplicated items. They classified the remaining items into seven cognitive domains then conducted panel meetings with clinicians and three Delphi surveys to select preliminary items for inclusion in the new screening test. After a feasibility study in ten healthy adults and five older adults with $\mathrm{MCl}$ the computer screening test was modified by removing items that did not differentiate between healthy controls and people with $\mathrm{MCl}$. The resulting mobile screening test system for screening mild cognitive impairment (mSTS-MCl) is administered on a tablet computer, scores from 0 to 28 with a higher score reflecting better cognitive function and reaction time is also measured. It can be administered in English or Korean, takes around 15 minutes to complete and the instruction manual in both languages is included with the test. The authors recruited 181 participants over the age of 65 years from community and welfare centres in South Korea. All were screened with the Seoul Neuropsychological Screening Battery by a blinded assessor with five years' clinical experience and were grouped into those with normal cognition $(\mathrm{N}=103)$ and those who met criteria for $\mathrm{MCl}(\mathrm{N}=74)$. All participants then underwent further testing with the Korean version of the Montreal Cognitive Assessment (MoCA-K), the Korean version of the Mini-Mental State Examination (MMSE-K) and the mSTS-MCI. The mSTS-MCI had good internal consistency and high test-retest validity. Scores on it were correlated with the MoCA-K but the area under the ROC for the mSTS-MCI was higher than for the MoCA-K, indicating better ability to 
discriminate between normal cognition and $\mathrm{MCl}$. The sensitivity and specificity of a cut-off score of $18 / 19$ on the mSTS-MCI was $97 \%$ and $93 \%$ respectively which were both higher than the sensitivity and specificity of the MoCA-K for detection of $\mathrm{MCl}$, although the scores for this version of the MoCA are lower than reported sensitivity and specificity for the English version of the MoCA. Overall, this paper validates a brief computerised screening tool for help in diagnosing $\mathrm{MCl}$ in a Korean population. It would be interesting to see how it performs in an English-speaking population, how it compares to the MMSE-K and to see further feasibility testing of incorporating it into routine clinic assessment as it could be helpful in terms of minimising the need for paper screening tools and would have the advantage of being automatically saved onto a computerised system.

Finally, on the theme of predicting progression from $\mathrm{MCl}$ to dementia, Beer et al (2018) recruited participants aged over 65 years from a clinical $(N=184)$ and population-based $(\mathrm{N}=435)$ sample, all of whom had a confirmed diagnosis of $\mathrm{MCl}$. They all underwent three trials of the Semantic Interference Test (SIT) which is described as a "cognitive stress test" that identifies very subtle deficits in early Alzheimer's disease (Loewenstein et al., 2004). The task involves identifying then recalling common everyday objects from one bag and then identifying and recalling objects from a second bag which are semantically related to objects in the first bag. Over four years, $23 \%$ of the clinical sample and $10 \%$ of the population-based sample had progressed to dementia. Using progression to dementia as a binary outcome, the authors tested sensitivity, specificity, positive and negative predictive value and area under the ROC to determine which score on the SIT best predicted progression to dementia. Classification accuracy of SIT thresholds ranged from $61.4 \%$ to $84.8 \%$ and AUC was $73.6 \%$ for the clinical sample and $72.9 \%$ for the population sample. The SIT therefore seems promising as a more fine-grained approach to detecting cognitive deficit in both clinical and population-based samples but as it is relatively complex, its utility in routine clinical testing still needs to be established and it may be more suited for more complex cases with more subtle deficits.

Overall these papers highlight the progress that has been made in terms of making diagnoses of $\mathrm{MCl}$ as robust and sensitive to change as possible. With further progress in this field we can move towards more timely and accurate diagnosis and be able to better predict progression to dementia in order to optimise interventions and reduce uncertainty for patients.

AMERICAN PSYCHIATRIC ASSOCIATION 2013. Diagnostic and statistical manual of mental disorders (DSM-5 $\left.{ }^{\circledR}\right)$, American Psychiatric Pub.

BEER, J.C., SNITZ, B.E., CHANG, C-C.H. \& LOEWENSTEIN, D.A. Does a cognitive stress test predict progression from mild cognitive impairment to dementia equally well in clinical versus population-based settings? International Psychogeriatrics, 2018, current issue.

COOPER, C., LI, R., LYKETSOS, C. \& LIVINGSTON, G. 2013. Treatment for mild cognitive impairment: systematic review. British Journal of Psychiatry, 203, 255-264.

GOMERSALL, T., SMITH, S. K., BLEWETT, C. \& ASTELL, A. 2017. 'It's definitely not Alzheimer's': Perceived benefits and drawbacks of a mild cognitive impairment diagnosis. British journal of health psychology, 22, 786-804. 
HEDMAN, A., KOTTORP, A., ALMKVIST, O \& NYGARD, L. Challenge levels of everyday technologies as perceived over five years by older adults with mild cognitive impairment. International Psychogeriatrics, 2018, current issue

KANE, R. L., BUTLER, M., FINK, H. A., BRASURE, M., DAVILA, H., DESAI, P., JUTKOWITZ, E., MCCREEDY, E., NELSON, V. A. \& MCCARTEN, J. R. 2017. Interventions to prevent age-related cognitive decline, mild cognitive impairment, and clinical Alzheimer's-type dementia.

KOROLEV, I. O., SYMONDS, L. L., BOZOKI, A. C. \& INITIATIVE, A. S. D. N. 2016. Predicting progression from mild cognitive impairment to Alzheimer's dementia using clinical, MRI, and plasma biomarkers via probabilistic pattern classification. PloS one, 11, e0138866.

LOEWENSTEIN, D. A., ACEVEDO, A., LUIS, C., CRUM, T., BARKER, W. W. \& DUARA, R. 2004. Semantic interference deficits and the detection of mild Alzheimer's disease and mild cognitive impairment without dementia. Journal of the International Neuropsychological Society, 10, 91-100.

MALINOWSKY, C., ALMKVIST, O., KOTTORP, A. \& NYGÅRD, L. 2010. Ability to manage everyday technology: a comparison of persons with dementia or mild cognitive impairment and older adults without cognitive impairment. Disability and rehabilitation: Assistive technology, 5, 462-469.

MANLY, J. J., TANG, M. X., SCHUPF, N., STERN, Y., VONSATTEL, J. P. G. \& MAYEUX, R. 2008. Frequency and course of mild cognitive impairment in a multiethnic community. Annals of Neurology: Official Journal of the American Neurological Association and the Child Neurology Society, 63, 494-506.

PARK, J-H., JUNG, M., KIM, J., PARK, H.Y., KIM, J-R. \& PARK, J-H. Validity of a novel computerized screening test system for mild cognitive impairment. International Psychoageriatrics, 2018, current issue.

ROBERTS, R. O., KNOPMAN, D. S., MIELKE, M. M., CHA, R. H., PANKRATZ, V. S., CHRISTIANSON, T. J., GEDA, Y. E., BOEVE, B. F., IVNIK, R. J. \& TANGALOS, E. G. 2013. Higher risk of progression to dementia in mild cognitive impairment cases who revert to normal. Neurology, 10.1212/WNL. 0000000000000055.

ROSENBERG, L., NYGÅRD, L. \& KOTTORP, A. 2009. Everyday technology use questionnaire: Psychometric evaluation of a new assessment of competence in technology use. OTJR: Occupation, Participation and Health, 29, 52-62. 